\title{
Infrared Radiative Corrections for Resonant Processes.
}

\author{
G. PANCHIERI \\ Laboratori Nazionali del CNEN - Frascati (Roma) \\ (Nuovo Cimento, $60 \mathrm{~A}, 321$ (1969))
}

Please add at the end of this paper the following acknowledgements:

I wish to thank Prof. B. Touschek for his continuous help and assistance throughout this work and Dr. E. EтIM for many helpful discussions. 